\title{
Lentivirus-mediated TGF-ß3, CTGF and TIMP1 gene transduction as a gene therapy for intervertebral disc degeneration in an in vivo rabbit model
}

\author{
YONG LIU, TAO YU, XUE-XIAO MA, HONG-FEI XIANG, YOU-GU HU and BO-HUA CHEN
}

Department of Orthopedic Surgery, The Affiliated Hospital of Qingdao University, Qingdao, Shandong 266003, P.R. China

Received November 24, 2014; Accepted January 12, 2016

DOI: $10.3892 / \mathrm{etm} .2016 .3063$

\begin{abstract}
The present study examined the effects of transforming growth factor (TGF)- $\beta 3$, connective tissue growth factor (CTGF) and tissue inhibitor of metalloproteinase 1 (TIMP1) gene transduction, using a lentiviral vector, on rabbit intervertebral disc degeneration in vivo, with the intention of investigating their potential use in gene therapy. A model of lumbar intervertebral disc degeneration was created by needle puncture into the annulus fibrosus of $15 \mathrm{New}$ Zealand white rabbits. Empty lentivirus or recombinant lentiviral plasmid lenti-TGF 33 -P2A-CTGF-T2A-TIMP1 was injected into degenerative lumbar intervertebral discs (representing the control and experimental groups, respectively), whilst untreated degenerative lumbar intervertebral discs served as the puncture group. After 16 and 20 weeks, magnetic resonance imaging (MRI) was conducted and the changes in intensity on micrographs of degenerative intervertebral discs were measured. The mRNA levels of aggrecan and type II collagen in nucleus pulposus tissue were determined by reverse transcription-polymerase chain reaction, and protein expression levels of type II collagen and aggrecan were determined by western blot analysis. MRI results indicated that intervertebral disc degeneration was ameliorated in the experimental group when compared with the control and the puncture group. Furthermore, the expression levels of type II collagen and aggrecan in the puncture and control groups were significantly lower than in the experimental group $(\mathrm{P}<0.05)$. In conclusion, lenti-TGFß3-P2A-CTGF-T2A-TIMP1 co-transduction can promote synthesis of aggrecan and type II collagen in degenerative intervertebral discs, thereby delaying intervertebral disc degeneration. These results indicate the potential of gene therapy in treatment of intervertebral disc degeneration.
\end{abstract}

Correspondence to: Dr Bo-Hua Chen, Department of Orthopedic Surgery, The Affiliated Hospital of Qingdao University, 16 Jiangsu Road, Qingdao, Shandong 266003, P.R. China

E-mail: chenbohuaqd@yeah.net

Key words: lentivirus, intervertebral disc degeneration, aggrecan, type II collagen

\section{Introduction}

Degenerative disc disease is a frequent cause of lower back pain. A previous study has demonstrated that $75-80 \%$ of people will experience lower back pain with prevalence ranging from 15 to $45 \%$. Severe disc degeneration is associated with a twofold increase in chronic low back pain (1). Current clinical treatment of this disease typically combines conservative and surgical treatment, although each method has been established to independently relieve symptoms. Surgery presents risks and may worsen the degeneration of adjacent tissue (2). Progress in molecular biology has enabled novel treatments, such as gene therapy, to emerge, and it may be possible to apply these in the treatment of disc degeneration. Reductions in proteoglycan and type II collagen levels are commonly associated with disc degeneration (2). Collagens provide form and tensile strength, whereas proteoglycans, through interactions with water, ensure tissue stiffness, viscoelasticity, and resistance to compression (2). A previous study has confirmed that single vector-mediated co-transduction of several genes significantly improves the effect of transgenic therapy (3). The authors of the present study have previously demonstrated that the adeno-associated virus 2 (AAV2)-connective tissue growth factor (CTGF)-tissue inhibitor of metalloproteinase 1 (TIMP1) vector is capable of significantly increasing the synthesis of type II collagen and proteoglycan in disc cells (4). Furthermore, transforming growth factor (TGF)- $\beta 3$ increased proliferation and the synthesis of extracellular matrix macromolecules; therefore, multi-gene therapy of intervertebral disc degeneration may be more effective. In the present study, a lentiviral plasmid encoding TGF- $\beta 3, \mathrm{CTGF}$ and TIMP1 was evaluated in a rabbit model of annulus fibrosus puncture-induced intervertebral disc degeneration. Plasmid was injected into the degenerative lumbar intervertebral discs in order to investigate whether it could delay the degeneration of the discs. Plasmids were synthesized using $2 \mathrm{~A}$ self-cleaving sequences to ligate the cDNA of TGF- $\beta 3$, CTGF and TIMP1 in a single open reading frame derived from lentiviral plasmids. Following transfection into 293 cells, reverse transcription-quantitative polymerase chain reaction (RT-PCR) and western blot analysis were performed to detect the mRNA and protein expression levels of TGF $\beta 3$, CTGF, TIMP1 at various time points following transfection. The recombinant plasmid, 
Table I. Primer sequences.

\begin{tabular}{llccc}
\hline Gene name & Accession number & $\begin{array}{c}\text { Forward } \\
\text { sequence }\left(5^{\prime} \rightarrow 3^{\prime}\right)\end{array}$ & $\begin{array}{c}\text { Reverse } \\
\text { sequence }\left(5^{\prime} \rightarrow 3^{\prime}\right)\end{array}$ & $\begin{array}{c}\text { Product } \\
\text { size }(\mathrm{bp})\end{array}$ \\
\hline $\begin{array}{l}\text { Type II collagen } \\
\text { Aggrecan }\end{array}$ & NM_001195671.1 & GGCTCCCAGAACATCACCTA & GATGACAGTCTTGCCCCACT & 198 \\
$\beta$-actin & NM_001101683.1 & TCCCTGGAGAAGAGCTACGA & GTGTTGGCGTACAGGTCCTT & 173 \\
\hline
\end{tabular}

lenti-TGF 33 -P2A-CTGF-T2A-TIMP1, was constructed successfully, providing a basic model for the packaging of virus and further study on therapy of intervertebral disc degeneration (5). The plasmid was subsequently injected into the degenerative lumbar intervertebral discs in order to investigate whether it could delay the degeneration of the discs.

\section{Materials and methods}

Animals. A total of 15 healthy, male New Zealand white rabbits (age, 1 year; weight, $2.5-3 \mathrm{~kg}$ ) were obtained from the Experimental Animal Center of the Affiliated Hospital of Qingdao University (Qingdao, China) and were housed in an animal care facility at $20-25^{\circ} \mathrm{C}$ (humidity, $40-50 \%$ ) with 12-h light/dark cycles and ad libitum access to food and water. Rabbits were divided into three groups: Experimental group, control group and puncture group ( $\mathrm{n}=5$ per group). Animal care and experimental protocols were approved by the Institutional Animal Care and Use Committee of the Affiliated Hospital of Qingdao University. New Zealand white rabbits were randomly divided amongst the experimental, control and puncture groups.

Puncture surgery and plasmid injection. An established animal model of intervertebral disc degeneration was created using the annulus fibrosus puncture technique, and this model was used in all the current experiments $(6,7)$. Each rabbit was anesthetized with an intramuscular injection of diazepam $(10 \mathrm{mg} / \mathrm{kg})$ and ketamine $(80 \mathrm{mg} / \mathrm{kg})$. Under sterile surgical conditions and general anesthesia, the dorsal skin was prepared aseptically at the central and left side; the spine was then exposed from an anterolateral, retroperitoneal approach. The L3-L4, L4-L5 and L5-L6 discs were sequentially punctured with a 16-gauge needle to a depth of $5 \mathrm{~mm}$. Four weeks after the puncture surgery, empty lentivirus or recombinant lentiviral plasmid lenti-TGF $33-P 2 A-C T G F-T 2 A-T I M P 1$ was injected into degenerative lumbar intervertebral discs (representing the control and experimental groups, respectively), whilst untreated degenerative lumbar intervertebral discs served as the puncture group. The surgical incisions were closed and standard postoperative care was performed. An intramuscular injection of 400,000 units penicillin was administered to all animals prior to and subsequent to surgery, and the animals were permitted to move freely in their cages following surgery.

mRNA expression of type II collagen and aggrecan. At 16 and 20 weeks after puncture surgery, intervertebral discs from each group were collected and homogenized in TRIzol reagent (Invitrogen; Thermo Fisher Scientific, Inc., Waltham, MA, USA) and total RNA was extracted according to the manufacturer's protocol. The concentration of total RNA was assessed using a spectrophotometer (DUR640; Beckman Coulter, Inc., Brea, CA, USA) and $1 \mu \mathrm{g}$ total RNA was transcribed into complementary (c)DNA using $0.5 \mu \mathrm{l}$ PrimeScript RTase (2-step), $1 \mu 1$ dNTPs (10 Mm), $1 \mu 1$ random primers $(20 \mu \mathrm{M}), 1 \mu \mathrm{g}$ template RNA, RNase-free $\mathrm{dH}_{2} \mathrm{O}$ up to $10 \mu \mathrm{l}$, $10 \mu 1$ PrimeScript buffer (5X), $4 \mu 1$ RNase inhibitor (40 U/ $\mu \mathrm{l})$, $5 \mu \mathrm{l}$ RNase-free $\mathrm{dH}_{2} \mathrm{O}, 5 \mu \mathrm{l}$ to a total volume of $20 \mu \mathrm{l}$. The reaction was performed at $42^{\circ} \mathrm{C}$ for $30 \mathrm{~min}, 95^{\circ} \mathrm{C}$ for $5 \mathrm{~min}$ and $4^{\circ} \mathrm{C}$ for $5 \mathrm{~min}$. The resultant cDNA was used in a PCR, performed with reagents from a Taq PCR Master Mix kit (Qiagen China Co., Ltd., Shanghai, China). The primers used for PCR amplification are listed in Table I, along with their GenBank accession numbers (National Institutes of Health, Bethesda, MD, USA). The optimal annealing temperatures were $54^{\circ} \mathrm{C}$ for type II collagen and aggrecan and $58^{\circ} \mathrm{C}$ for the internal control gene $\beta$-actin. Thermal cycling was performed using an Eppendorf Mastercyler (535025012; Eppendorf AG, Hamburg, Germany). Initial denaturation of the reaction mixture was performed at $95^{\circ} \mathrm{C}$ for $5 \mathrm{~min}$. PCR amplification was conducted using the following cycling conditions: Denaturation, $98^{\circ} \mathrm{C}$ for $30 \mathrm{sec}$; annealing at the aforementioned temperature for $30 \mathrm{sec}$; and extension, $72^{\circ} \mathrm{C}$ for $20 \mathrm{sec}$, for 37 cycles, as previously described (8). This was followed by a final extension step at $72^{\circ} \mathrm{C}$ for $10 \mathrm{~min}$. PCR products were then separated by agarose gel electrophoresis using agarose gel that had been stained with ethidium bromide (E8751; Sigma-Aldrich) prior to analysis (Sigma-Aldrich, St. Louis, MO, USA). Band intensities of the target genes were measured by densitometry using an Vilber Lourmat image analysis system (version 3.1; Vilber Lourmat GmbH, Eberhardzell, Germany). mRNA levels were normalized and expressed as a ratio of the band intensity of the sample to that of the internal reference gene $\beta$-actin (Fig. 1). Experiments were performed $\geq 3$ times.

Western blotting. After 16 and 20 weeks, the nuclei pulposi from each group were collected, the cells were lysed using $3 \mathrm{ml} / \mathrm{g}$ radioimmunoprecipitation assay lysate (R0278; Sigma-Aldrich) into the nucleus pulposus tissue and protein was extracted by ultrasonication in mammalian protein extraction reagent and protease inhibitor (BioVision, Inc., Hangzhou, China). The mixture was incubated on ice for $2 \mathrm{~h}$, then centrifuged at $4^{\circ} \mathrm{C}$ for $10 \mathrm{~min}$ at $\sim 20,000 \mathrm{x} \mathrm{g}$. The supernatant was stored at $-70^{\circ} \mathrm{C}$. Total protein was separated by sodium dodecyl sulfate-polyacrylamide gel electrophoresis and the separated proteins were transferred by electroblotting 
A
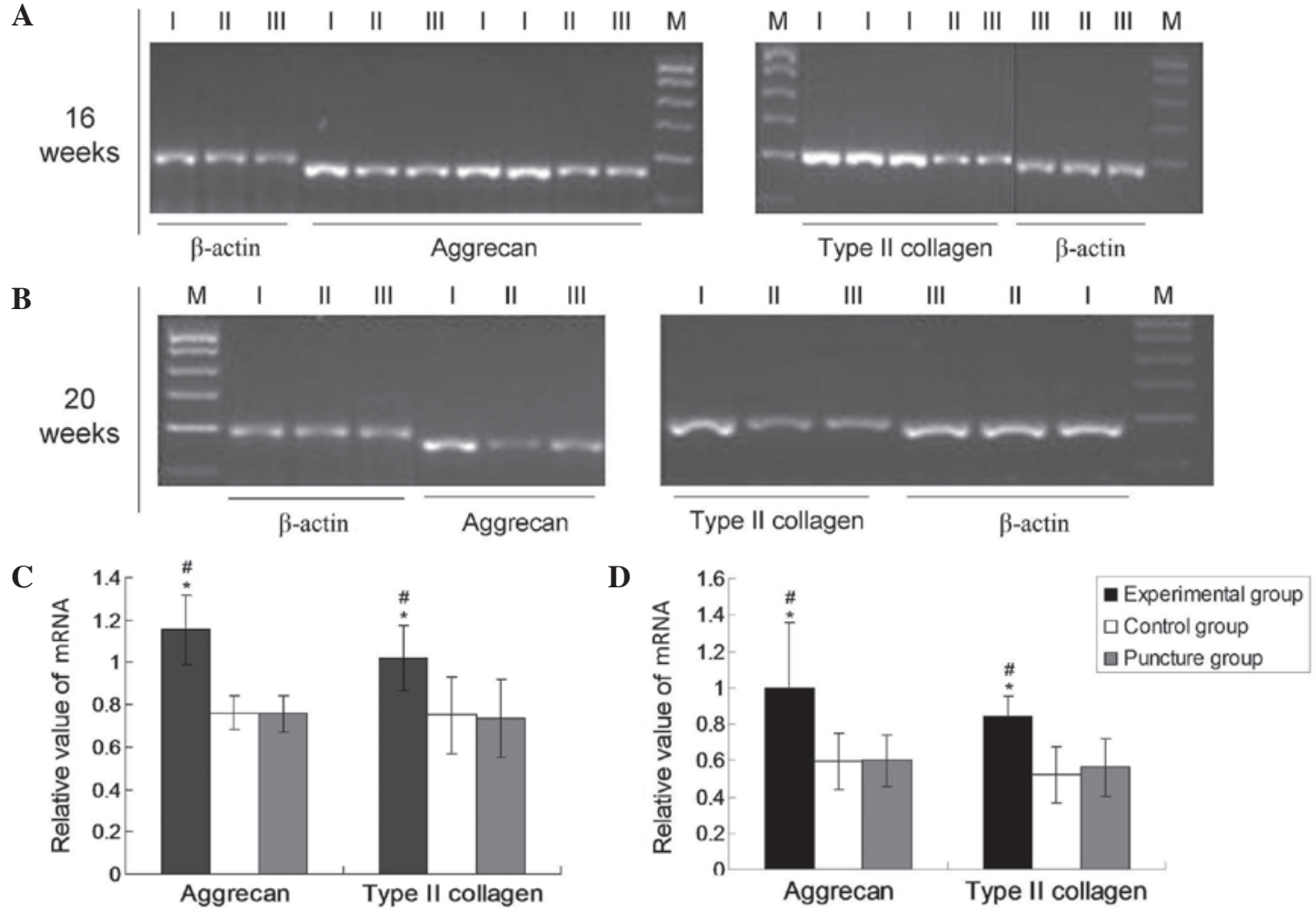

Figure 1. mRNA expression of aggrecan and type II collagen. Reverse transcription-polymerase chain reaction results for aggrecan and type II collagen mRNA at (A) 16 and (B) 20 weeks, and the expression levels at (C) 16 and (D) 20 weeks as determined by densitometric analysis relative to $\beta$-actin. Data are presented as the mean \pm standard deviation. "P $<0.05$ vs. the control group; ${ }^{~} \mathrm{P}<0.05$ vs. the puncture group. Lanes: $\mathrm{M}$, Marker; I, experimental group; II, control group; III, puncture group.

A

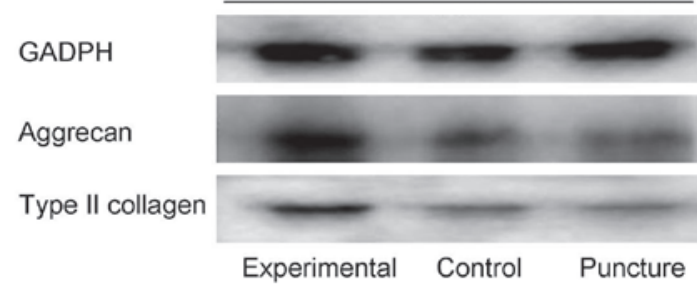

C

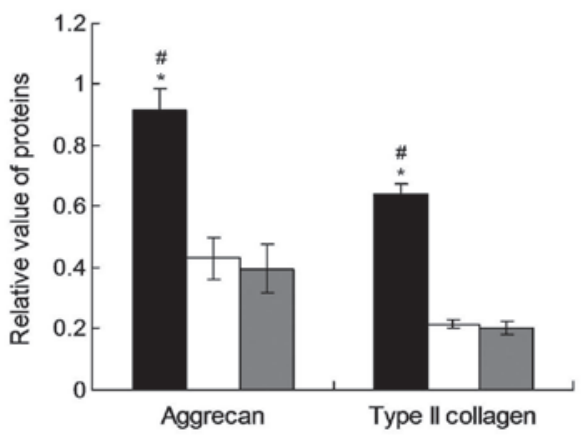

B

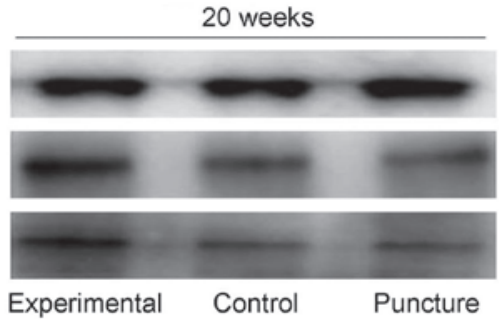

D

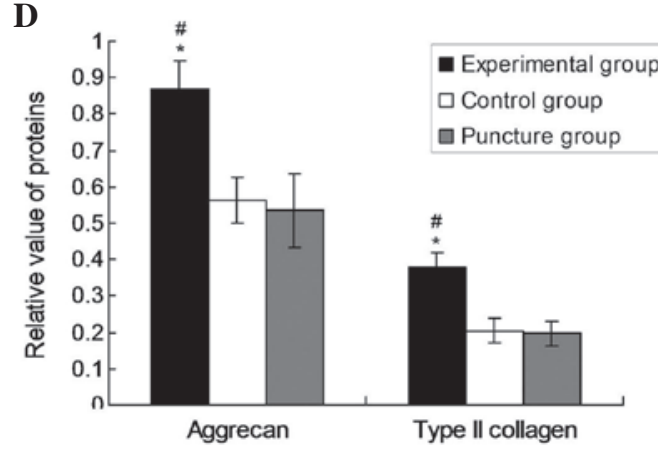

Figure 2. Protein expression of aggrecan and type II collagen at 16 weeks and 20 weeks. Representative western blots for aggrecan and type II collagen at (A) 16 and (B) 20 weeks, and (C and D) the expression levels at (C) 16 and (D) 20 weeks as determined by densitometric analysis. Data are presented as the mean \pm standard deviation. ${ }^{*} \mathrm{P}<0.05$ vs. the control group; ${ }^{~} \mathrm{P}<0.05$ vs. the puncture group. GAPDH, glyceraldehyde-3-phosphate.

to a polyvinylidene difluoride membrane. The membrane was then incubated at room temperature for $2 \mathrm{~h}$ with primary antibodies from Bioss, Inc. (Beijing, China), as follows: Monoclonal rabbit anti-collagen-II (1:100; bs-0709R), monoclonal rabbit anti-aggrecan (1:100; bs-1223R) and monoclonal mouse anti-glyceraldehyde-3-phosphate dehydrogenase (GAPDH; 1:500; bsm-0978M). Following three washes with phosphate-buffered saline with Tween 20 (PBST; BioVision, Inc.), the membrane was incubated with horseradish peroxidase-labeled secondary antibody $(1: 3,000$; 


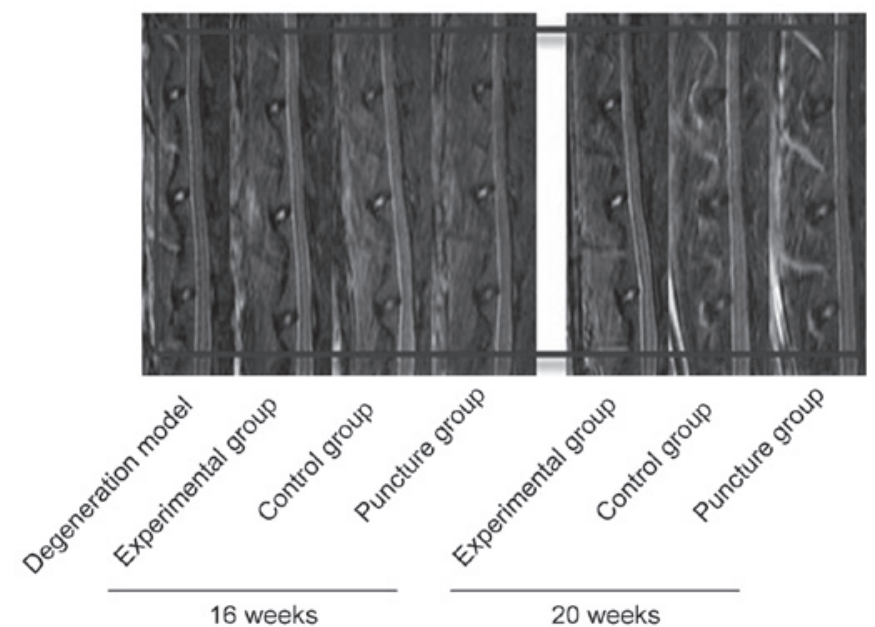

Figure 3. T2-weighted midsagittal lumbar magnetic resonance images of L3-4, L4-5 and L5-6 vertebrae at 16 and 20 weeks.

A

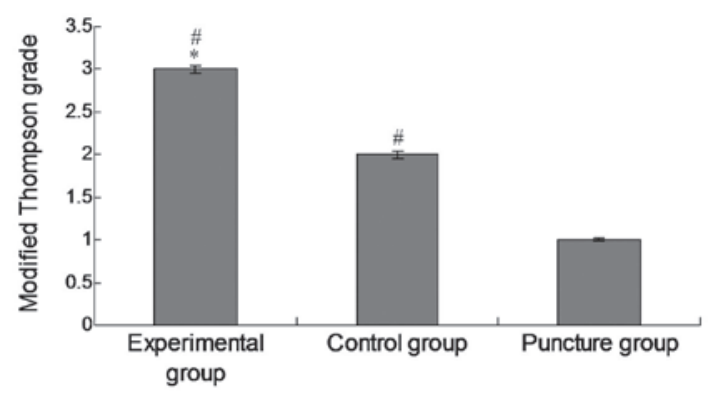

B

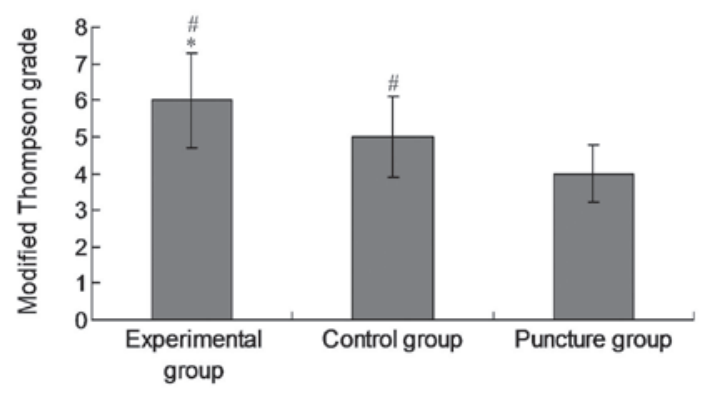

Figure 4. Modified Thompson grades of intervertebral disc degeneration. Modified Thompson grades were evaluated at (A) 16 and (B) 20 weeks, respectively. Data are presented as the mean \pm standard deviation. ${ }^{*} \mathrm{P}<0.05$ vs. the control group; ${ }^{\#} \mathrm{P}<0.05$ vs. the puncture group.

bs-0295G-HRP; Bioss, Inc.) in PBST for $1 \mathrm{~h}$ at room temperature. Following removal of the secondary antibody, a bioluminescent substrate (EMD Millipore, Billerica, MA, USA) was added and the membrane was developed using a IS4000MM chemiluminescence imaging system (Kodak, Rochester, NY, USA). Bands were quantified using Quantity One software (Bio-Rad Laboratories, Inc.). GAPDH served as an internal reference protein and each experiment was repeated 12 times.

Magnetic resonance imaging (MRI). Under general anesthesia of $10 \mathrm{mg} / \mathrm{kg}$ ketamine and $0.5 \mathrm{mg} / \mathrm{kg}$ diazepam (i.m.), each rabbit was scanned by MRI (Magnetom Trio 3T; Siemens AG, Munich, Germany) to evaluate the degeneration of the intervertebral discs at 16 and 20 weeks. All images were analyzed using the modified Thompson method, as previously described (9), which categorizes T2-weighted images according to four levels: Level I, normal high intensity; level II, mild hypointensity; level III, moderate hypointensity; and level IV, severe hypointensity.

Statistical analysis. All values are presented as the mean \pm standard deviation. Statistical analyses were performed using SPSS software, version 15.0 (SPSS, Inc., Chicago, IL, USA). A one-way analysis of variance was used to compare groups differences between the groups. MRI data were analyzed using a random sum test. $\mathrm{P}<0.05$ was considered to indicate a statistically significant difference.

\section{Results}

Expression of aggrecan and type II collagen $m R N A$. The mRNA expression levels of aggrecan and type II collagen were normalized to that of the endogenous reference gene, $\beta$-actin. As shown in Fig. 1, the mRNA expression levels of collagen II and aggrecan were significantly upregulated in the experimental group after 16 and 20 weeks compared with the levels in the puncture and control groups $(\mathrm{P}<0.05)$. However, no significant differences were revealed in the expression levels of aggrecan and collagen II between the puncture and control groups at these time points $(\mathrm{P}>0.05)$. In the experimental group at 16 and 20 weeks, the aggrecan values were $1.0993 \pm 0.15032$ and $1.0031 \pm 0.54409$, respectively, and the type II collagen values were $1.0168 \pm 0.11753$ and $0.8696 \pm 0.15402$, respectively. In the puncture group at 16 and 20 weeks, the aggrecan values were $0.6848 \pm 0.11576$ and $0.6017 \pm 0.31039$, respectively, and the type II collagen values were $0.6636 \pm 0.18730$ and $0.5402 \pm 0.11997$, respectively. In the control group, the aggrecan values at 16 and 20 weeks were $0.6992 \pm 0.10144$ and $0.5932 \pm 0.33179$, respectively and the type II collagen values were $0.6897 \pm 0.17477$ and $0.5247 \pm 0.12953$, respectively. These results demonstrate that lenti-TGF $\beta 3$-CTGF-TIMP1 co-transduction significantly promoted the expression of aggrecan and type II collagen.

Protein expression of aggrecan and type II collagen. Densitometric values from western blot analyses were evaluated by computerized laser densitometry and normalized to that of the endogenous reference protein GAPDH. As shown in Fig. 2, the levels of aggrecan and type II collagen protein in the experimental group were significantly increased compared with those in the control and puncture groups after 16 and 20 weeks $(\mathrm{P}<0.05)$. However, there was no significant difference in the levels of aggrecan and type II collagen protein between the puncture group and the control group $(\mathrm{P}>0.05)$. In the experimental group at 16 and 20 weeks, the aggrecan values were $0.1188 \pm 0.00536$ and $0.1160 \pm 0.01208$, respectively and the type II collagen values were $0.1259 \pm 0.01124$ and $0.1101 \pm 0.01039$, respectively. In the puncture group at 16 and 20 weeks, the aggrecan values were $0.0767 \pm 0.00918$ and $0.0548 \pm 0.01045$, respectively, and the type II collagen values were $0.0866 \pm 0.1118$ and $0.0740 \pm 0.00937$, respectively. In the control group, the aggrecan values at 16 and 20 weeks were $0.0759 \pm 0.00810$ and $0.0599 \pm 0.01124$, respectively and the type II collagen 
values were $0.0900 \pm 0.00945$ and $0.0732 \pm 0.01596$, respectively. These data indicate that lenti-TGF 33 -CTGF-TIMP1 co-transduction significantly promoted the protein expression of aggrecan and type II collagen.

MRI. Comparisons of the degeneration of intervertebral discs among the three groups were made by MRI scanning at 16 and 20 weeks. Degeneration progressively increased within this time period in the control and puncture groups (Fig. 3); however, no statistically significant difference was observed between these two control groups at $16(n=6)$ or 20 weeks $(n=9)(P>0.05)$. By contrast, degeneration in the experimental group was significantly reduced compared with that in the two control groups $(\mathrm{P}<0.05)$.

Modified Thompson grades. In order to evaluate the therapeutic effects of the experimental treatment on intervertebral disc degeneration, the modified Thompson grade of each group was examined. The modified Thompson grade of the experimental group was significantly increased $(\mathrm{P}<0.05)$ compared with those of the control and puncture groups at 16 (Fig. 4A) and 20 weeks (Fig. 4B), which is indicative of attenuated degeneration following the administration of lenti-TGFß3-CTGF-TIMP1.

\section{Discussion}

Intervertebral disc degeneration typically results from the increased activity of matrix metalloproteinases and decreased secretion of proteoglycan and type II collagen $(10,11)$. The degenerative disc undergoes complex biochemical changes that involve a progressive loss of proteoglycan content, leading to dehydration of the nucleus pulposus (12).

Previous studies have revealed that TGF- $\beta 3$ can promote the synthesis of type II collagen by fibroblastic cells in the degenerating intervertebral disc $(13,14)$, whilst the expression of TGF- $\beta 3$ is downregulated in degenerating in intervertebral discs. Nakanishi et al (15) reported that CTGF enhances proteoglycan and type II collagen expression in chondrocytes and inhibits apoptosis. Furthermore, CTGF can inhibit matrix degradation (16) and induce angiogenesis through the expression of tissue inhibitor of metalloproteinases 1 (TIMP1). TIMPs are anticatabolic growth factors that prevent matrix metalloproteinases from enzymatically cleaving proteoglycans (17). TIMP1 inhibits the enzymatic activity of matrix metalloproteinase 3 in the intervertebral disc (18). Furthermore, Bachmeier et al (17) found that matrix metalloproteinases exacerbate the degeneration of the intervertebral disc, whilst their inhibitors can block degenerative processes.

A number of previous studies have reported promising results using a biological approach in the treatment of degenerative intervertebral discs $(19,20)$. The adenoviral vector AAV2-CTGF-TIMP1 was constructed in 2010, with subsequent successful use in animal experiments (4); single and double vector-mediated expression of common genes has since been confirmed to significantly increase the effectiveness of transgenic therapy (3). However, in order to implement such improvements in transgenic therapy, vector use must be optimized. The lentiviral expression system is considered to be one of the most effective gene therapy vectors available, being able to transfect almost all mammalian cell types (including mitotic and amitotic cells) and integrating foreign genes into host chromosomes, making it a more stable and efficient expression system than the transient expression achieved using an adenoviral vector (21-24). Following alteration, the lentiviral vector can accommodate up to $\sim 10 \mathrm{~kb}$ of exogenous genes, which is an improvement on the limited capacity of the adenoviral vector, providing favorable conditions for the implementation of a multiple gene expression system. In 2012, a lenti-TGFß3-CTGF-TIMP1 gene expression plasmid was successfully constructed (5); in the present study, an intervertebral disc degeneration model was generated, and this vector was used to transduce multiple genes into the degenerative intervertebral disc. The present study aimed to observe the downstream consequences of this lentiviral vector on proteoglycan and type II collagen, and thereby to elucidate the mechanism of action of this therapy during degeneration of the intervertebral disc. The present study confirmed that lenti-TGF $\beta 3$-CTGF-TIMP1 increased the synthesis of proteoglycan and type II collagen (demonstrated by reverse transcription-PCR and western blotting). Furthermore, significant differences observed using MRI examination provided indirect evidence that lenti-TGF $\beta 3$-CTGF-TIMP1 delayed intervertebral disc degeneration.

The current study demonstrated the successful use of a lentiviral vector as a multiple gene expression plasmid in vivo. This was a novel approach, providing a rationale for subsequent development of gene therapy, for instance through use of alternative candidate genes in treatment of intervertebral disc degeneration.

In the present experiments, a model of intervertebral disc degeneration was created using the annulus fibrosus puncture technique. This differs from the complex process involved in clinical cases, but the intervention measures used in this model indicate the effectiveness and feasibility of multiple gene treatment. Compared with the control and puncture groups, mRNA and protein expression levels of aggrecan and type II collagen were markedly increased in the experimental group. As compared with the AAV2-CTGF-TIMP1 gene transduction, aggrecan and type II collagen mRNA expression was 1.26- and 1.89-fold higher at 16 weeks, and at 20 weeks mRNA expression of aggrecan and type II collagen (lenti-TGF 33 -CTGF-TIMP1) was 2.11- and 2.54-fold higher, as compared the expression detected at 24 weeks (AAV2-CTGF-TIMP1) (4), demonstrating that lenti-TGF $\beta 3$-CTGF-TIMP1 gene transduction can have a stabilizing role in vivo, which endures for 20 weeks. However, the potential of its use in complete reversal of disc degeneration requires additional study. It should be noted that the effects of the experimental intervention were assessed at an early stage of intervertebral disc degeneration in the present study, and the effects of the treatment are likely to differ if it is used at later stages of the disc degeneration process. In subsequent studies, it is therefore important to examine the later stages of disc degeneration.

The present experiments confirm the effectiveness of co-transduction of multiple genes in the treatment of intervertebral disc degeneration. It is also important to note that no apparent side effects were observed in the rabbits in the present study, side effects may remain a risk in the clinical setting. For instance, there is a risk of injury when puncturing the intervertebral disc for the 
injection of drugs. The suitability of injection doses, long-term effectiveness and potential side effects all require additional study prior to the use of gene therapy clinically; however, the present results demonstrate promising potential.

In conclusion, the present experimental results confirm that co-transduction with multiple genes using the lentiviral plasmid lenti-TGF 33 -P2A-CTGF-T2A-TIMP1 can promote the synthesis of aggrecan and type II collagen and delay degeneration of the intervertebral disc in an animal model. Although this experimental approach has its limitations, the results demonstrate the potential of this approach in the clinical treatment of intervertebral disc degeneration.

\section{Acknowledgements}

The present study was funded by the National Natural Science Foundation (grant no. 81171758).

\section{References}

1. Takatalo J, Karppinen J, Niinimäki J, Tainela S, Näyhä S, Mutanen P, Sequeiros RB, Kylönen E and Tervonen O: Does lumbar disc degeneration on magnetic resonance imaging associate with low back symptom severity in young Finnish adults? Spine 36: 2180-2189, 2011.

2. Park P, Garton HJ, Gala VC, Hoff JT and McGillicuddy JE: Adjacent segment disease after lumbar or lumbosacral fusion: Review of the literature. Spine (Phila Pa 1976) 29: 1938-1944, 2004

3. Cui K, Zhou X, Luo J, Feng J, Zheng M, Huang D, Jiang J, Chen X, Wei Y, Li J and Yang L: Dual gene transfer of bFGF and PDGF in a single plasmid for the treatment of myocardial infarction. Exp Ther Med 7: 691-696, 2014.

4. Liu Y, Kong J, Chen BH and Hu YG: Combined expression of CTGF and tissue inhibitor of metalloprotease-1 promotes synthesis of proteoglycan and collagen type II in rhesus monkey lumbar intervertebral disc cells in vitro. Chin Med J (Engl) 123: 2082-2087, 2010.

5. Jiang F, Yue B, Ma XX, Zhang GQ, Hu YG and Chen BH: Construction and detection of lentiviral plasmids containing human transforming growth factor beta 3 , connective tissue growth factor and tissue inhibitor of metalloproteinases 1 gene. Zhongguo Zuzhi Gongcheng Yanjiu Yu Linchuang Kangfu 16: 699-703, 2012 (In Chinese).

6. Sobajima S, Kompel JF, Kim JS, Wallach CJ, Robertson DD, Vogt MT, Kang JD and Gilbertson LG: A slowly progressive and reproducible animal model of intervertebral disc degeneration characterized by MRI, X-ray and histology. Spine (Phila Pa 1976) 30: 15-24, 2005.

7. Masuda K, Aota Y, Muehleman C, Imai Y, Okuma M, Thonar EJ, Andersson GB and An HS: A novel rabbit model of mild, reproducible disc degeneration by an anulus needle puncture: Correlation between the degree of disc injury and radiological and histological appearances of disc degeneration. Spine (Phila Pa 1976) 30: 5-14, 2005.

8. Tsirimonaki E, Fedonidis C, Pneumaticos SG, Tragas AA, Michalopoulos I and Mangoura D: PKCe signalling activates ERK1/2, and regulates aggrecan, ADAMTS5, and miR377 gene expression in human nucleus pulposus cells. PLoS One 8: e82045, 2013.
9. Yang H, Wu J, Liu J, Ebraheim M, Castillo S, Liu X, Tang T and Ebrahein NA: Transplanted mesenchymal stem cells with pure fibrinous gelatin-transforming growth factor-betal decrease rabbit intervertebral disc degeneration. Spine J 10: 802-810, 2010.

10. Antoniou J, Steffen T, Nelson F, Winterbottom N, Hollander AP, Polle RA, Aebi M and Alini M: The human lumbar intervertebral disc: Evidence for changes in the biosynthesis and denaturation of the extracellular matrix with growth, maturation, ageing, and degeneration. J Clin Invest 98: 996-1003, 1996.

11. Leung VY, Chan D and Cheung KM: Regeneration of intervertebral disc by mesenchymal stem cells: Potentials, limitations, and future direction. Eur Spine J 15: 406-413, 2006.

12. Pearce RH, Grimmer BJ and Adams ME: Degeneration and the chemical composition of the human lumbar intervertebral disc. J Orthop Res 5: 198-205, 1987.

13. ten Dijke P, Hansen P, Iwata KK, Pieler C and Foulkes JG: Identification of another member of the transforming growth factor type beta gene family. Proc Natl Acad Sci USA 85: 4715-4719, 1988.

14. Risbud MV, Di Martino A, Guttapalli A, Seghatoleslami R, Denaro V, Vaccaro AR, Albert TJ and Shapiro IM: Toward an optimum system for intervertebral disc organ culture: TGF-beta 3 enhances nucleus pulposus and anulus fibrosus survival and function through modulation of TGF-beta-R expression and ERK signaling. Spine (Phila Pa 1976) 31: 884-890, 2006

15. Nakanishi T, Nishida T, Shimo T, Kobayashi K, Kubo T, Tamatani T, Tezuka K and Takigawa M: Effects of CTGF/Hcs24, a product of a hypertrophic chondrocyte-specific gene, on the proliferation and differentiation of chondrocytes in culture. Endocrinology 141: 264-273, 2000.

16. McLennan SV, Wang XY, Moreno V, Yue DK and Twigg SM: Connective tissue growth factor mediates high glucose effects on matrix degradation through tissue inhibitor of matrix metalloproteinase type 1: Implications for diabetic nephropathy. Endocrinology 145: 5646-5655, 2004.

17. Bachmeier BE, Nerlich A, Mittermaier N, Weiler C, Lumenta C, Wuertz $\mathrm{K}$ and Boos N: Matrix metalloproteinase expression levels suggest distinct enzyme roles during lumbar disc herniation and degeneration. Eur Spine J 18: 1573-1586, 2009.

18. Yang H, Wu J, Liu J, Ebraheim M, Castillo S, Liu X, Tang T and Ebraheim NA: Transplanted mesenchymal stem cells with pure fibrinous gelatin-transforming growth factor-betal decrease rabbit intervertebral disc degeneration. Spine J 10: 802-810, 2010.

19. Zhao H, Ni CF, Huang J, Zhao SM, Gu WW, Jiang H, Chen L and Tan TS: Effects of bone cement on intervertebral disc degeneration. Exp Ther Med 7: 963-969, 2014.

20. Masuda K: Biological repair of the degenerated intervertebral disc by the injection of growth factors. Eur Spine J 17: S441-S451, 2008.

21. Miyoshi H, Smith KA, Mosier DE, Verma IM and Toebett BE: Transduction of human $\mathrm{CD}^{+} 4^{+}$cells that mediate long-term engraftment of NOD/SCID mice by HIV vectors. Science 283: 682-686, 1999.

22. Naldini L, Blomer U, Gallay P, Ory D, Mulligan R, Gage FH, Verma IM and Frono D: In vivo gene delivery and stable transduction of nondividing cells by a lentiviral vector. Science 272 : 263-267, 1996.

23. Santoni de Sio F and Naldini L: Short-term culture of human CD34+ cells for lentiviral gene transfer. Methods Mol Biol 506: 59-70, 2009.

24. Zamule SM, Strom SC and Omiecinski CJ: Preservation of hepatic phenotype in lentiviral-transduced primary human hepatocytes. Chem Biol Interact 173: 179-186, 2008. 